\title{
Evaluación Sociointercultural para Proyectos de Inversión en Comunidades Indígenas wixarikas
}

\author{
José G. Vargas-Hernández* \\ Ernesto Guerra García* \\ María Eugenia Meza Hernández ${ }^{* * *}$
}

Se analizan aspectos de la problemática que se presenta en la evaluación social de proyectos de inversión para las comunidades indígenas wixarikas (huicholes). Los proyectos en este contexto hacen particularmente compleja la evaluación, ya que en la perspectiva socioeconómica con la que se evalúa entra en juego la inconmensurabilidad de los asuntos de carácter social e intercultural que no se pueden pasar por alto. Se abordan las interrogantes que han surgido en la elaboración de este tipo de proyecto y se presenta un marco teórico para la propuesta metodológica de evaluación sociointercultural.

\section{Introducción}

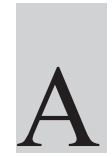
1 elaborar proyectos de inversión para la implementación de energías alternativas en las comunidades wixarikas (hicholes) en México en el año 2010, se detectó que había una serie de aspectos a discutir en la teoría

\footnotetext{
* Universidad de Guadalajara. Centro Universitario de Ciencias Económico Administrativas. jvargas2006@gmail.com. Ph. D. (Administración Pública) por Columbia States University y Ph. D. (Economía) por Keele University. MBA por Pacific States University. Miembro del Sistema Nacional de Investigadores.

** Universidad Autónoma Indígena de México. drguerragarcia@gmail.com. Doctor en Enseñanza Superior por el Centro de Investigación y Docencia en Humanidades del Estado de Morelos. Maestro en Economía por la Universidad Autónoma de Nuevo León. Miembro del Sistema Nacional de Investigadores.
}

*** Universidad Autónoma Indígena de México. uaim_mmeza@yahoo.com. Maestra en Educación Social por la Universidad Autónoma Indígena de México. Facilitadora Educativa de la Carrera de Sociología Rural. 
Palabras clave: evaluación social de proyectos de inversión, evaluación sociointercultural, comunidades indígenas, wixarikas.

This paper analyzes aspects of the problem that occurs in the social evaluation of investment projects for indigenous communities' Wixarikas (Huichols). A project in this context make particularly complex the evaluation. On the socio-economic perspective with which it is evaluated co- mes into play the incommensurability of social and intercultural issues that cannot be ignored. It is addressed the questions that have arisen in the development of this type of project and presents a theoretical framework for the methodological proposal of socio-cultural evaluation.

Keywords: Social evaluation of investment projects, socio-intercultural evaluation, indigenous communities, Wixarikas.

de la evaluación social de proyectos de inversión cuando éstos son aplicados en un contexto indígena. Estos proyectos buscan mejorar las condiciones de los wixarikas y de otras comunidades indígenas a través de impulsar la infraestructura básica que además posibilite la generación de proyectos con enfoques y principios propios acordes con las culturas y las lógicas económicas de las etnias que participan, así como de su racionalidad social y ambiental, en especial la relación que guardan con la madre tierra ${ }^{1}$.

En las comunidades wixarikas, el hecho de valorar la posibilidad de llevar el servicio eléctrico a través de energías alternativas presenta de antemano externalidades que pueden considerarse negativas para su cultura, pues este servicio implicaría un mayor uso de televisores, radios y demás medios de comunicación y abriría la posibilidad de ampliar un proceso aculturador que a pesar de los beneficios, los efectos negativos podrían ser aun más indeseables. Pero el simple hecho de querer ayudar a los wixarikas como parte de una política gubernamental pudiera tener implicaciones racistas al colocar a la cultura mestiza nacional por encima de la de ellos. No es un asunto sencillo, la muestra está en que a pesar del alto interés por esta cultura, en las últimas décadas no se ha podido contribuir a mejorar significativamente el bienestar económico y material de esta etnia ${ }^{2}$.

Es evidente que la cultura no indígena tiene un peso mayor y que las decisiones tendrán un sesgo particular en este sentido, pero a través de una serie de

\footnotetext{
${ }^{1}$ Gómez González, G., Gómez Calderón, E. X. and Gómez Calderón, Y. (2008). Perspectiva de los agronegocios en el desarrollo indígena: caso Querétaro. Ra Ximahi, (4) 003, 4-14.

${ }^{2}$ Wiegand, P. and Fikes, J. (2004). "Sensacionalismo y etnografía, el caso delos huicholes de Jalisco”, en Relaciones, vol. 25, núm. 098, México: Colegio de Michoacán., p. 54.
} 
asuntos éticos en las políticas públicas se podrían tomar en cuenta cualificaciones del mundo indígena para tratar de sopesar sus intereses. Por ejemplo, a diferencia del mundo no indígena, para los campesinos huicholes lo productivo y lo religioso está tan íntimamente ligado con la vida económica y social que aparentemente presentan una falta de interés para la adopción y adaptación tecnológica ${ }^{3}$.

Esto no significa que los wixarikas se encuentren aislados de la sociedad mestiza, la persistencia de su cultura y comunidad puede ser explicada a través de procesos de identificación de cara al mundo, pero la especificidad de su etnia se debe, en parte, a la creativa integración de lo que no es su cultura ${ }^{4}$.

La compleja madeja para el análisis de proyectos en estos contextos inicia con la consideración de que en la evaluación social, menciona Fontaine ${ }^{5}$, las externalidades permiten entender la factibilidad de impulsar un proyecto sin fines de lucro y en un contexto socointercultural, éstas van en múltiples sentidos y tendrían que analizarse en lo intrasocial, lo intracultural y lo intercultural ${ }^{6}$.

Al introducir esta perspectiva metodológica de análisis que hemos llamado sociointercultural ${ }^{7}$ en la evaluación social se abre un área de investigación para generar modelos que describan las categorías a considerar en este tipo de ambientes.

Lo anterior implica que la evaluación se realice además en forma 'multicriterial', es decir, reconocer que al tratar problemas complejos como los que se presentan en las etnorregiones se necesita tener en cuenta la inconmensurabilidad social, cultural, intercultural e intracultural presente en estos. Esta inconmensurabilidad se refiere a la presencia de múltiples valores legítimos en la

\footnotetext{
${ }^{3}$ Torres Contreras, J. J. (2000), Tierras magras y politicas equivocadas en el sistema productivo huichol, caso Santa Catarina, municipio de Mezquitic, Jalisco, Espiral, (7), 019. 162-163.

${ }^{4}$ Florentine Beimborn, M. and Romandía Peñaflor, A. (2009). Emigración y continuidad cultural de los wixaritari. Breve reflexión sobre una relación ambigua, Liminar, Estudios Sociales y Humanísticos, (7) 2.13.

${ }^{5}$ Fontaine, E. (2008). La evaluación privada y social de proyectos: el rol del Estado. Panorama socioeconómico, (26) 036. 8-17.

${ }^{6}$ Guerra García, E. (2004), La sociointerculturalidad y la educación indígena. En Sandoval Forero, E. y Baeza, M. A. (coord..). Cuestión étnica, culturas, construcción de identidades, México: UAIM, ALAS, El Caracol.

${ }^{7}$ Idem.
} 
sociedad y en las culturas (variados puntos de vista y en conflicto) que conllevan no sólo a la necesidad de involucrar en el proceso de toma de decisiones a los agentes afectados, sino de entender las políticas del Estado para el efecto ${ }^{8}$.

Pero ¿cómo realizar una evaluación sociointercultural de un proyecto de inversión en una comunidad indígena? o más específicamente ¿Cuáles son las categorías a considerar en este tipo de evaluaciones? Estas cuestiones han sido analizadas para el caso mencionado y se describen brevemente en el presente artículo.

\section{Desarrollo \\ Evaluación de proyectos de inversión}

Se entiende como proyecto de inversión a la formulación de una intervención en un medio para estudiar una problemática existente y el análisis de la factibilidad de lograr un cambio deseado al menos por alguna parte de la sociedad; el proyecto de inversión es aquel donde se plasma con claridad y detalle lo que se desea lograr y además cómo lograrlo; permite justificar la intervención desde distintos puntos de vista para dar o no solución a una problemática ${ }^{9}$. En cualquier caso, aun cuando el objetivo sea privado, la evaluación debe considerarse una forma de investigación social

...aplicada, sistemática, planificada y dirigida, en que se apoya un juicio acerca del mérito y el valor de los diferentes componentes de un programa, de forma tal que sirva como base o guía para una toma de decisiones racional e inteligente entre cursos de acción ${ }^{10}$.

Sin hacer a un lado los aspectos técnicos económicos y financieros, el hecho de que muchas de las externalidades sean difíciles de cuantificar dificulta la evaluación en general.

\footnotetext{
${ }^{8}$ Vargas Isaza, O. L. (2005). La evaluación multicriterio social y su aporte a la conservación de bosques. Revista Facultad Nacional de Agronomía - Medellin. (58) 1, 1-22.

${ }^{9}$ Andia Valencia, W. (2010). Proyectos de inversión, un enfoque diferente de análisis. Industrial Data, (13) 1.28-29.

${ }^{10}$ Matos Basó, R. (2005). Enfoques de evaluación de programas sociales: análisis comparativo. Revista de Ciencias Sociales (Ve), (11) 002. 23.
} 
Evaluar es uno de los conceptos más difíciles de abordar en amientes sociointerculturales ya que generalmente no es posible la implementación de una métrica válida y aceptada por todos los interesados. Además, los aspectos que comúnmente son considerados de validez universal son cuestionados ante la presencia de otras formas de ver el mundo. Evaluar significa entonces para este caso esclarecer todas las dudas que la operación de un proyecto pudiera tener antes de que éste sea aplicado desde el polirelativismo y el multicriterio mencionado. Las incertidumbres que se presentan se deben en gran parte por los problemas que implica la información sociointercultural y las dificultades para la prescripción y la determinación del resultado final ${ }^{11}$.

\section{El enfogue de la economía social}

La crisis de los modelos de desarrollo ha permitido la visibilidad de algunas formas ancestrales de entender la economía y el surgimiento de innovaciones que se han venido a llamar economía del tercer sector, economía solidaria, economía del trueque, economía popular o economía social ${ }^{12}$.

En realidad toda economía es social, sin embargo cuando el enfoque es el privado se dejan de lado todas las consideraciones de los otros actores que participan en el total económico ${ }^{13}$. La finalidad no es agregar una variable endógena más sino reconocer preponderantemente las dimensiones sociales de la economía ${ }^{14}$. El objetivo de la economía social no es el lucro; se trata de un modelo orientado al bienestar de grupos y colectividades ${ }^{15}$, de esta forma un proyecto de energía alternativa en estas comunidades asegura la sustentabilidad, aun cuando el costo de la inversión es elevado y aparentemente no se tenga un resultado financiero positivo.

\footnotetext{
${ }^{11}$ Arroyave Loaiza, G. (1994). Análisis de sensibilidad de los proyectos de inversión en salud. Salud Pública de México, (36) 003, 318-27.

${ }^{12}$ Bastidas Delgado, O. y Richer, M. (2001). Economía social y economía solidaria: intento de definición, Cayapa, (1) 001. 1.

${ }^{13}$ Idem, p. 2.

${ }^{14}$ Izquierdo Server, R. (2009). Responsabilidad social de las empresas, crisis y economía social. CIRIEC-España, Revista de Economía Pública, Social y Cooperativa. 65. p. 5.

${ }^{15}$ Pujol, J. (2003).La economía social en Cataluña. CIRIEC- España Revista de Economía Pública, Social y Cooperativa. 047. p. 36.
} 
Además, la economía social se difunde a través de un proceso de reconocimiento de las pobres circunstancias en las que se encuentra una comunidad indígena y de la deuda que por más de 500 años tiene la sociedad mexicana para este sector ${ }^{16}$.

La economía indígena se compone de prácticas ancestrales de adaptación a un medio determinado que se componen de los siguientes elementos a) la producción que determina un paisaje determinado de acuerdo con la forma particular de apropiación del territorio de cada pueblo indígena trabajadas con técnicas tradicionales, b) la distribución, donde operan mecanismos diferentes a la intermediación como reciprocidad y redistribución c) el consumo, caracterizado por las formas de igualación d) la organización del trabajo y e) la tierra, que es vista desde una cosmovisión diferente al de la propiedad individual ${ }^{17}$. Los elementos precolombinos, que se componen de prácticas ancestrales de adaptación a un medio determinado, donde no interviene el dinero, corresponden a una economía que puede ser llamada tradicional ${ }^{18}$, pero existen muchos elementos que se han creado a partir de la relación con el mundo no indígena, quizás la relación económica más antigua de estos últimos ha sido el comercio.

Para que lo anterior se lleve a cabo es necesario el real y verdadero reconocimiento de las organizaciones sociales en este caso del gobierno, de las comunidades y de los pueblos indígenas. De esta forma las políticas públicas son de gran relevancia para la evaluación en este tipo de contextos ${ }^{19}$.

\section{La evaluación social}

Un proyecto de inversión social busca cumplir con objetivos sociales a través de metas gubernamentales o alternativas, empleadas por programas de apoyo ${ }^{20}$.

\footnotetext{
${ }^{16}$ Bastidas Delgado, O. y Richer, M. Op. cit., p. 2.

${ }^{17}$ Lugo, D. (2007). Economía indígena y estrategias de reproducción en el grupo indígena Warao. en Cayapa. (7) 013. 60.

${ }^{18}$ Idem.

${ }^{19}$ Huot, G. and Bissiéres, D. (2006). El grupo (Chantier) de economía social y los sectores de la economía social en Québec. Cayapa, (6) 011. 124..

${ }^{20}$ Matos Basó, R. Op. cit.
} 
En el caso que nos ocupa es de resaltar que las zonas indígenas en México presentan yuxtaposiciones entre los usos e intereses que el suelo implica en la cosmovisión de sus pueblos y las actividades preponderantemente económicas de los no indígenas ${ }^{21}$. Los ejemplos más recientes han implicado la lucha de algunas etnias contra el uso y explotación minera de algunos intereses privados sobre los usos que los pueblos indígenas quieren hacer de su suelo ${ }^{22}$. Entonces la diferencia respecto a las otras evaluaciones es que los beneficios, los costos y las externalidades deben observarse desde las diferentes perspectivas al mismo tiempo. Es decir, en los proyectos interculturales no es suficiente realizar la formulación y la evaluación desde una sola perspectiva, sino que es necesario poner sobre la mesa todos los criterios y puntos de vista de las culturas que participan.

La problemática que se presenta es que en la valoración social existen elementos perceptibles por una comunidad como perjuicio que son difíciles de cuantificar o de generar una ponderación en unidades monetarias. De aquí que el desarrollo de este tipo de proyectos tiene preferentemente un enfoque cualitativo.

Pero esto no quiere decir que su desarrollo sea menos valioso. Al contrario, las discusiones desde una perspectiva sociointercultural conllevan a generar nuevos constructos que permiten entender lo que sucede en una realidad social concreta.

\section{Las externalidades}

Los proyectos de investigación social siempre conllevan una serie de aristas referente al manejo de las externalidades que no sólo no han sido resueltas, sino que se van planteando en la medida en que se encuentran en la práctica. Las externalidades se producen cuando las actividades sociales o económicas de un grupo de personas tienen un impacto sobre otro o sobre la naturaleza y dicho

\footnotetext{
${ }^{21}$ Korsbaek, L. (2009), Los peligros de la comunidad indígena y sus defensas. Ra Ximahi. (5) 003. 373-85. ${ }^{22}$ Saliba, F. (30 de diciembre de 2011). En México, los indígenas huicholes no quieren las minas de oro y plata, Le monde, París Francia; La Jornada (11 de marzo de 2011). Minera canadiense pone en riesgo a pueblos indígenas. La Jornada; Zapateando (27 de marzo de 2012), Indígenas marchan para la libertad de Patishtán y contra minas y presas. Zapateando. Recuperado de http://zapateando.wordpress.com/2012/03/27/indigenas-marchan-para-la-libertad-de-patishtan-y-contra-minas-y-presas-accion-urgente-por-la-libertad-de-alberto-patishtan/
} 
impacto no es tomado en cuenta de manera adecuada por el primer grupo ${ }^{23}$.

En el tratamiento de las externalidades es importante mencionar que desde el enfoque social calcular los costos del efecto negativo y luego tratar de pagarlo de manera correctiva no es precisamente lo óptimo ${ }^{24}$; es decir, internalizar las externalidades no es la mejor filosofía en la evaluación social, pues cuando los grupos son de diferentes culturas existen situaciones de carácter intercultural que deben ser resueltas de manera preventiva.

\section{Economia y gestión de los recursos naturales}

La importancia de este tipo de proyectos se incrementa al considerar que a las comunidades indígenas rurales se les ha asignado la tarea de ser proveedoras de recursos a las zonas urbanas y se les ha atribuido la responsabilidad de preservar el equilibrio ambiental ${ }^{25}$.

Además una característica adicional que tienen este tipo de proyectos de energía alternativa en las comunidades indígenas es que a la par se abordan aspectos de economía y gestión de los recursos naturales. Al respecto hay que resaltar que el interés por las Fuentes de Energía Nuevas y Renovables (FENR) se ha debido a la crisis de energía que cada vez más se acentúa ${ }^{26}$. Dentro de esta disciplina se encuentra la economía ecológica, que a diferencia de la teoría económica convencional, su objetivo no es la búsqueda de la eficiencia, de la rentabilidad y del crecimiento en términos puramente monetarios, sino de tratar de hacer compatible la sustentabilidad del capital natural ${ }^{27}$.

\footnotetext{
${ }^{23}$ Jaime, A. y Tinoco López, R. O. (2006), Métodos de valuación de externalidades ambientales provocadas por obras de ingeniería, Ingeniería e investigación y tecnología. (7) 002.105.

${ }^{24}$ Fontaine, E. La evaluación..., Op. cit., p. 13.

${ }^{25}$ Mozas Moral, A. y Bernal Jurado, E. (2006). Desarrollo territorial y economía social. CIREC-España Revista de Economía Pública, Social y Cooperativa, 055.127.

${ }^{26}$ Rodríguez-Murcia, H. (2008). Desarrollo de la energía solar en Colombia y sus perspectivas. Revista de Ingeniería, 28. 88.

${ }^{27}$ Domínguez Torreiro, M. (2004). El papel de la fisiocracia en nuestros días: una reflexión sobre el análisis económico de los recursos naturales y el medio ambiente. Revista Galega de Economía, (13), 001-002. 8 .
} 
Lo que sí es evidente es que la dependencia humana de los ecosistemas se aprecia de manera evidente en economías de subsistencia ligadas al medio natural, donde las comunidades humanas, como las indígenas, toman directamente de los ecosistemas sólo lo que necesitan para vivir; de esto, las comunidades wixarikas tienen una gran sabiduría. El reconocimiento de este hecho, implica asumir que el desarrollo económico y social dependerá en el mediano y largo plazo no sólo del adecuado mantenimiento de los sistemas ecológicos que lo sustentan y que constituyen el capital natural del planeta sino también del respeto y de la atención que pongamos en la culturas indígenas, de las que tenemos mucho que aprender ${ }^{28}$.

Las cuestiones relacionadas con los recursos naturales son analizadas tanto desde la perspectiva económica como desde el marco institucional con sus reglas, deberes y obligaciones, formales e informales ${ }^{29}$; además deben ser consideradas las formas de relación que cada cultura tiene con la naturaleza.

\section{La incorporación de La dimensión ambiental en el análisis de proyectos}

En este tipo de proyectos en comunidades indígenas rurales es difícil dejar de lado la evaluación de impacto ambiental, que consiste en la identificación, análisis y valoración de efectos de los proyectos sobre su entorno, natural y social, desde el polirelativismo y el multicriterio, aun cuando no necesariamente éstos estén expresados en unidades monetarias.

Para la evaluación del impacto ambiental de un proyecto sobre el medio económico es posible acotar que desde el momento de su construcción y posterior puesta en marcha, éste estará influyendo en el medio donde se instalará, por los efectos que produciría sobre las actividades naturales, humanas y económicas existentes y futuras durante su período de operación y hasta la etapa de abandono.

\section{Desarrolloobuen vivir}

${ }^{28}$ Gómez, B. E and de Groot, R. (2007). Capital natural y funciones de los ecosistemas: explorando las bases ecológicas de la economía. Ecosistemas, (16) 003. 5-6.

${ }^{29}$ Dominguez Torreiro, M. Op. cit., p. 6-7. 
Otro elemento a considerar en la evaluación de proyectos en las comunidades indígenas es que en América Latina está en marcha una renovación de la crítica al desarrollo convencional bajo un proceso que ofrece varias particularidades y que aporta otro enfoque a la evaluación social. Lo importante aquí y como se trata de la evaluación en comunidades wixarikas es que las posturas del 'buen vivir' recuperan visiones ancladas en los conocimientos saberes propios de pueblos indígenas. Las posturas del buen vivir cuestionan al desarrollo con su filosofía de progreso y que en la práctica a significado una relación adversa con la naturaleza.

El buen vivir es un concepto de política pública en construcción, pero en general recupera la idea de una buena vida, del bienestar en un sentido más amplio y en el caso de la economía social y de la evaluación social provee como criterio general que una comunidad viva bien, sin esperar el progreso a costa de la devastación de los recursos naturales.

De lo anterior se destaca que la evaluación de un proyecto es diferente si se enmarca dentro de alguna de las políticas de desarrollo o dentro de la que se presenta con el enfoque del buen vivir. Las políticas públicas son determinantes en la orientación de los trabajos de evaluación social.

\section{Las comunidades wixarikas}

Para la cultura huichol, también llamada wixarika, hacerse sabio significa saber de la naturaleza ${ }^{30}$. Para esta cultura el mundo mestizo es un altermundo que coexiste de manera mítica con el suyo ${ }^{31}$.

La palabra 'huichol' proviene de 'hueitzolme', un territorio ubicado actualmente en Nayarit; su lengua pertenece a la rama totorame de la familia uto-azteca del sur ${ }^{32}$. Los wixarikas habitan en la región Huicot que comprende aproximadamente doscientas cincuenta mil hectáreas que comparten los estados de Nayarit, Durango, Jalisco y Zacatecas. Esta zona está ubicada en la Sierra Madre Occiden-

\footnotetext{
${ }^{30}$ Iturrioz, citado por Juránková, M. (2007). El perfil comunicativo de los huicholes que viven en la ciudad. Comunicación y Sociedad. 007.150.

${ }^{31}$ Durín, S. (2005). Sacrificio de res y competencia por el espacio entre los wixaritari (huicholes), Alteridades, (15), 029. 91.

${ }^{32}$ Wiegand, P. and Fikes, J. Op. cit., 51-52.
} 
tal en una amplia franja denominada el 'Gran Nayar', pero el peso que el desierto localizado en San Luis Potosí tiene para ellos es determinante para su cultura ${ }^{33}$.

Este tipo de emigración en los los wixarikas permite entender en mayor perspectiva las dinámicas de su cultura en su intensiva interacción con 'el otro'34.

En general es una región pobre con caminos son de terracería y veredas, la luz eléctrica es sumamente escasa ya que los problemas de acceso a este territorio dificulta la instalación de servicios y comunicaciones ${ }^{35}$. Además de los wixarikas habitan en esta región otros grupos étnicos además de los mestizos: los coras, los tepehuanos, los tepecanos y los mexicaneros que en total congregan 56,614 indígenas ${ }^{36}$.

El pueblo wixarika ha sentado sus actividades agrícolas desde cuando menos hace 900 años ${ }^{37}$; tradicionalmente residen en tres comunidades, San Sebastián, Santa Catarina y San Andrés, que junto con Tuxpan y Guadalupe de Ocotán constituyen las cinco unidades territoriales políticas que fueron constituidas desde la época de la Corona española en el siglo XVIII ${ }^{38}$.

Según el último Censo de Población y Vivienda del Instituto Nacional de Estadística, Geografía e Informática (INEGI), en estas regiones habitan 44,788 hablantes de huichol con una edad mayor a los cinco años, de los cuales 22,129 son hombres y 22,659 mujeres (INEGI, 2010). Según el INEGI (2011) esta lengua se encuentra en el lugar 22 en volumen de hablantes, antes del chontal y después del chatino; pero es uno de los grupos con un mayor porcentaje de monolingüismo en México ${ }^{39}$.

La organización productiva de los grupos en esta etnorregión se ha concentrado en actividades primarias de índole agropecuario; la cría de ganado bovino,

\footnotetext{
${ }^{33}$ Porras Carrillo, E. (2006). Algunos aspectos de las relaciones entre el desierto y los huicholes. Culturales. (2) 003. 34.

${ }^{34}$ Florentine Beimborn y Romandía Peñaflor. Op. cit., p. 15.

${ }^{35}$ Barrera, R. O. (2002). Consideraciones geomorfológicas sobre la Sierra Madre Occidental en el norte de Jalisco, México, Investigaciones Geográficas, 048, 45.

${ }^{36}$ Guízar Vázquez, F. (2009). Wixaritari (huicholes) y mestizos: análisis heurístico sobre un conflicto intergrupal, Indiana, 26, 171.

${ }^{37}$ Tetreault, D. V. and Lucio López, C. F. (2011). Jalisco: pueblos indígenas y regiones de alto valor biológico. Espiral. (18) 51. 170.

${ }^{38}$ Wiegand y Fikes. Op. cit., p. 51.

${ }^{39}$ Juránková, M. Op. cit., p.149.
} 
tanto de los wixarikas como de los demás grupos étnicos es la actividad más relevante. La agricultura de temporal y la explotación forestal también han tomado relevancia en los últimos años ${ }^{40}$. El prolongado conflicto intra e intercultural se hace más crudo frente a la colonización de rancheros mestizos que han tenido el soporte del Estado para avanzar en el proceso etnofágico que resulta de las asimetrías de los grupos indígenas y no indígenas. El hecho es que el territorio wixarika ha sido reclamado cada día con mayor insistencia desde la época de la colonia y en nuestros días ese reclamo se realiza de maneras más sofisticadas por el grupo mestizo; la lucha actual no solamente es en la yuxtaposición de las regiones mineras con las zonas sagradas, sino que el grupo mestizo utiliza la educación, religión y tecnología, entre otros, para penetrar y modificar su mundo.

Estas y otras consideraciones sociointerculturales de la vida de los wixarikas no pueden ser dejadas de lado en la evaluación de un proyecto de inversión.

\section{Propuestadeevaluación sociointercultural}

En esta complejidad descrita la propuesta para la evaluación sociointercultural radica en estructurar las categorías de análisis de acuerdo con las macroesferas y las microesferas en las categorías que corresponden a los asuntos a) intrasociales, b) los asuntos intraculturales y c) los asuntos interculturales. En la figura 1 se presenta un esquema en alusión a lo anterior.

Figura 1. Esquema para el análisis sociointercultural

\begin{tabular}{|c|c|}
\hline DIMENSIONES & $\begin{array}{lll}\text { INTRASOCIAL } & \text { INTRACULTURAL } & \text { INTERCULTURAL }\end{array}$ \\
\hline $\begin{array}{c}\text { MACROESFERA } \\
\text { Politica, social, economica }\end{array}$ & \multirow{2}{*}{ SOCIOINTERCULTURAL } \\
\hline $\begin{array}{l}\text { MICROESFERA } \\
\text { Género, clase y etnia }\end{array}$ & \\
\hline
\end{tabular}

Fuente: construcción de los autores

\section{La evaluación desde las macroesferas}

${ }^{40}$ Guízar Vásquez, F. Op. cit., p.177. 
Para evaluar un proyecto como el que se presenta es necesario que se tome en cuenta las macroesferas política, social y económica. Para el caso de las comunidades wixarikas los criterios máximos provienen de a) las tendencias en las políticas públicas, ya sean del desarrollo o del buen vivir, las que implican a su vez lo que el Estado desea hacer con los pobres y con los marginados, que en la mayoría de los casos convergen en generar las sinergias necesarias en los más necesitados; b) las tendencias ecologistas a nivel mundial y a nivel nacional que favorecen las tecnologías alternativas y evitar aquellas que se suman al calentamiento global y c) el interculturalismo, lo que el Estado desea hacer para con los grupos étnicos que conforman la nación, es decir, en qué medida y cómo es que se orientan los esfuerzos hacia los pueblos indígenas. Quizás estas tendencias en las políticas públicas son las más importantes en la consideración de la evaluación de cualquier proyecto de inversión.

\section{La evaluación desde las microesferas}

Puesto que se habla de proyectos específicos, la evaluación debe considerar las manifestaciones de los diferentes agentes implicados, los gobiernos locales, el pueblo wixarika en este caso y la sociedad mestiza que se encuentra en la vecindad y que posiblemente reciban también externalidades de los proyectos. En este caso es importante considerar otros aspectos de la especificidad de la comunidad que participa, que pueden también orientar la decisión final; por ejemplo la constitución demográfica en la wixarika es relevante.

\section{Laevaluación desde lo intrasocial}

En esta categoría se incluye el análisis de costos, beneficios y externalidades que tienen que ver más con los asuntos de la sociedad independientemente de las culturas y etnias que participan. En este caso, el uso sustentable de las tecnologías, las políticas de atención a la pobreza independientemente del grupo étnico a la que pertenezca la población en este Estado, entre otras, pertenecen a la evaluación intrasocial. El uso de la energía alternativa en las comunidades evita utilizar energías dañinas; aquí el problema radica en valorar el posible costo o el beneficio ecológico. Esto es debido a que la contaminación se considera una externalidad negativa generada por los procesos de producción y de consumo, en este caso de la energía 
eléctrica ${ }^{41}$. Por otro lado, la inclusión de los intereses de las futuras generaciones trae a las comunidades rurales e indígenas oportunidades de ciertos incentivos provenientes de las políticas globales para la mitigación y adaptabilidad al cambio climático a través del mecanismo del desarrollo limpio de energías ${ }^{42}$.

La presencia de proyectos de corte sustentable es uno de los aspectos intrasociales que hacen compleja esta evaluación, pues el valor del uso de la energía alternativa es más que significativo, independientemente de las culturas involucradas.

\section{Laevaluación desde lo intracultural}

En la práctica resulta que las comunidades indígenas no son un todo uniforme, pues mientras algunas personas se niegan a tener los beneficios de la energía alternativa debido a que observan ciertos peligros de aculturación, otras los prefieren para aplicarlo en los usos domésticos y de producción que les darían una mejor forma de vida. Es decir, no todos los wixarikas se manifiestan en consenso sobre los proyectos de intervención.

En el caso de los mestizos es lo mismo, no todos están de acuerdo con ayudar a una comunidad wixarika, sobre todo si hay otras - indígenas o no - que también requieren los beneficios. La tecnología utilizada por los campesinos wixarikas normalmente se encuentra integrada a su estructura y dinámica sociocultural y es a partir de su percepción del medio que instrumentan un sistema técnico culturalmente específico, de tal forma que toda innovación tecnológica trastoca su modo de vida su manera de ver el mundo y sus valores ${ }^{43}$.

De aquí surgen muchos cuestionamientos que a final de cuentas se encuentran ligados con externalidades. ¿Cómo afecta o podría trastocar el uso de la ener-

\footnotetext{
${ }^{41}$ Reyes Gil, Rosa E., Galván Rico, Luís E. y Aguilar Serra, Mauricio (2005), El precio de la contaminación como herramienta económica e instrumento de política ambiental. Interciencia, (30) 007. 436.

${ }^{42}$ Pinto Silbato, F. (2004). Energías renovables y desarrollo sostenible en zonas rurales de Colombia. El caso de la Vereda Carrizal en Sutamarchán, Cuadernos de Desarrollo Rural, 053. 124.

${ }^{43}$ Berrueta Soriano, V. M., Limón Aguirre, F., Fernández Zayas, José L. y Soto Pinto, M. L. (2003), Participación campesina en el diseño y construcción un secador solar para café, Agrociencia, (37) 001. 95.
} 
gía alternativa al sistema técnico cultural de los wixarikas?, ¿cómo cambiaría esta tecnología su modo de vida, su manera de ver el mundo y sus valores? ¿ permite esta tecnología un mayor arraigo de los habitantes y de sus valores culturales?

Al llegar la energía eléctrica a la comunidad, algunas de las personas que pensaban emigrar ya no lo harían debido a que posiblemente los satisfactores pudieran ser suficientes para que las personas permanezcan, posiblemente alterando su tradición migratoria. La llegada de la energía va ligada también con el uso de medios de comunicación y con estos los procesos de aculturación se incrementan. ¿De qué forma se darían estos procesos? ¿cuánto se valora el desplazamiento de una lengua en una cultura y en la sociedad? Son preguntas que no se pueden resolver de manera simple.

\section{La evaluación desde lo intercultural}

La evaluación de los proyectos interculturales debe ser entendida relacionándola con estrategias políticas contextualizadas. No puede pensarse en la interculturalidad desde una lógica instrumental, que propicia la extensión o universalización de un modelo transcultural con supuestas buenas intenciones. Tampoco se pueden trasladar los mismos criterios utilizados en contextos diferentes. En la evaluación están presentes, las formaciones, estructuras y resistencias; las relaciones de desigualdad social y la lucha para transformarlas.

De esta forma una política pública orientada a la expansión de la energía en medios rurales indígenas no siempre es deseable, debido a los dinámicos procesos de aculturación que generalmente se tienen en las relaciones interculturales ${ }^{44}$.

Aquí se tiene que evaluar las externalidades que existen entre las culturas cuando el proyecto intensifica las relaciones interculturales. Los efectos aculturadores deben ser analizados, sobre todo los de de la sociedad no indígena sobre los wixarikas, la perdida de los valores culturales, tales como lengua, costumbres y en general la influencia en su cosmovisión.

Pero ¿cómo evaluar las externalidades cuando los criterios son inconmensurables? Por ejemplo, al evaluar cualquier proyecto de inversión se tiene que

${ }^{44}$ Pinto Silbato, F. Op. cit., p. 123. 
observar la posibilidad de contaminación de la tierra. El problema radica en que, para la cultura wixarika la tierra es sagrada y no debe ser trastocada. En resumen la solución se torna imposible. Dejar que otros decidan por ellos no es equitativo ni justo, por lo que se está precisamente en una encrucijada más.

\section{Conclusión}

Se concluye aquí que es necesario abrir investigaciones en la línea de la evaluación sociointercultural en el contexto indígena, para abordar con más profundidad cada una de las externalidades planteadas. La evaluación sociointercultural de proyectos de inversión es una metodología de investigación social que se enmarca dentro de la aplicación de las políticas públicas, que abarca mucho más que la aplicación de técnicas cuantitativas de corte financiero y de la perspectiva privada. De manera transversal al análisis de las macro y microesferas se propone estudiar los aspectos intrasociales, intraculturales e interculturales característicos de las sociedades pluriculturales.

Como se explicó, el análisis de las macroesferas parte de preceptos de la economía social y considera los aspectos propios de la economía indígena en la que se contraponen las teorías de desarrollo con las emergentes propuestas del buen vivir. En esta metodología, queda en evidencia que los asuntos técnico financieros se ven reducidos ante la necesidad de profundizar en los análisis cualitativos de las externalidades.

La complejidad de la evaluación se acrecienta cuando los proyectos en cuestión se refieren a energías alternativas que se enmarcan a su vez en economías ecológicas de los recursos naturales, donde la idea de la sustentabilidad marca de por si una diferencia significativa en las formas de hacer evaluación en los proyectos sociales de inversión.

En resumen, desde la perspectiva de la economía sociointercultural, los proyectos de energía alternativa en comunidades wixarikas no podrían esperar el pago pecuniario de la inversión por una generación de campesinos en su mayoría, ya que su nivel económico no lo permitiría. Sin embargo, la inversión se justifica ya que se promovería el desarrollo social y económico de la comunidad, pero además si ésta se hiciera a través del uso de una energía renovable, generaría externalidades positivas al mundo y al futuro de la humanidad. Este último valor es el que justifica plenamente el proyecto. 